Pacific

Journal of

Mathematics

ON THE STRUCTURE OF THE VALUE SEMIGROUP OF A VALUATION

C. Galindo 


\section{ON THE STRUCTURE OF THE VALUE SEMIGROUP OF A VALUATION}

\section{Galindo}

Let $v$ be a valuation of the quotient field of a noetherian local domain $R$. Assume that $v$ is centered at $R$. This paper studies the structure of the value semigroup of $v, S$. Ideals defining toric varieties can be defined from the graded algebra $K[T]$ of cancellative commutative finitely generated semigroups such that $T \cap(-T)=\{0\}$. The value semigroup of a valuation $S$ need not be finitely generated but we prove that $S \cap(-S)=\{0\}$ and so, the study in this paper can also be seen as a generalization to infinite dimension of that of toric varieties.

In this paper, we prove that $K[S]$ can be regarded as a module over an infinitely dimensional polynomial ring $A_{v}$. We show a minimal graded resolution of $K[S]$ as $A_{v}$-module and we give an explicit method to obtain the syzygies of $K[S]$ as $A_{v}$-module. Finally, it is shown that free resolutions of $K[S]$ as $A_{v}$-module can be obtained from certain cell complexes related to the lattice associated to the kernel of the map $A_{v} \rightarrow$ $K[S]$.

\section{Introduction.}

Let $(R, m)$ be a noetherian local domain. Denote by $F$ its quotient field and by $K$ its residue field. A valuation of $F$ centered at $R$ (a valuation in the sequel) is a mapping $v$ of the multiplicative group of $F$ onto a totally ordered commutative group $G$, such that the following conditions are satisfied:

1. $v(x y)=v(x)+v(y)$;

2. $v(x+y) \geq \min \{v(x), v(y)\}$;

3. $v$ is nonnegative on $R$ and strictly positive on $m$.

$G$ is called to be the value group of the valuation $v$. The set $S:=\{v(f) \mid f \in$ $R \backslash\{0\}\}$ is a commutative semigroup called the value semigroup of the valuation $v$. Note that when $\operatorname{dim} R=2$, the most known case, $S$ contains a lot of information about $v$. Our aim, in this paper, is to study the structure of $S$ by extending methods of the toric geometry.

Section 2 of the paper provides some basic properties of the semigroup $S$. S need not be finitely generated. However, it satisfies an interesting 
property: $S$ is combinatorially finite, i.e., the number of decompositions of any element in $S$ as a finite sum of others in $S$ is finite. When $S$ is finitely generated, this property is equivalent to $S \cap(-S)=\{0\}$. There exists an extensive literature $[\mathbf{5}, \mathbf{2}, \mathbf{1}, \mathbf{4}]$, which studies the graded algebra $K[S]$ of cancellative commutative finitely generated semigroups $S$ such that $S \cap$ $(-S)=\{0\}$ (this study includes ideals defining toric varieties). Therefore, we devote Section 3 to extend to the $S$-graded algebra $K[S]$, now $S$ being the value semigroup of a valuation, the ideas of the toric case. Essentially we use the fact that $S$ is combinatorially finite, so our study can also be seen as a generalization to infinite dimension of that of toric varieties.

Subsection 3.1 deals with $K[S]$ regarded as a module over an infinitely dimensional, in general, polynomial ring $A_{v}$. Both $K[S]$ and $A_{v}$ are $S$-graded. We construct a minimal graded resolution of $K[S]$ as $A_{v}$-module and prove that an explicit isomorphism can be given between the (finitely dimensional) vector space of degree $\alpha$ syzygies $(\alpha \in S)$ and the vector space of augmented homology of a simplicial complex $\triangle_{\alpha}$ introduced in [3]. Furthermore, we give a combinatoric method, adapting the one in [4], that allows us to obtain $\widetilde{H}_{i}\left(\triangle_{\alpha}\right)$ explicitly from vector space complexes associated to directed graphs. These directed graphs are associated to partitions of certain finite subsets of a generating set of $S$. The most interesting situation arises when the partitions are induced by the value subsemigroup of $S$ defined by a subring $T$ of $R$ such that $v$ is also centered at $T$.

Subsection 3.2 is divided in two parts, their nexus being the fact that the kernel $I_{0}$ of the mapping $A_{v} \rightarrow K[S]$, which gives to $K[S]$ structure of $A_{v}$-module, is spanned by binomials. In 3.2 .1 , we characterize, by means of a graphic condition, when a set of binomials constitutes a minimal homogeneous generating set of $I_{0}$. On the other hand, $I_{0}$ is spanned by a set of binomials satisfying that the difference between their exponents is in a lattice $L . L$ is intimately related to the value group $G$ of the valuation (see the beginning of Section 3). In 3.2.2, we associate to $L$ an $A_{v}$-module $M_{L}$ and we show how suitable cell complexes on minimal generating sets of the $A_{v}$-module $M_{L}$ give rise to free resolutions of $M_{L}$, called cellular ones, and how some of these resolutions allow us to get free resolutions of $K[S]$ as $A_{v}$-module.

\section{The value semigroup of a valuation.}

Let $S$ be a commutative semigroup with a zero element. $S$ is said to be a cancellative semigroup if it satisfies a cancellative law, i.e., if $\alpha, \beta, \gamma \in S$ and $\alpha+\beta=\alpha+\gamma$ then $\beta=\gamma$. Associated to $S$, we can consider an abelian group $G(S)$ and a semigroup homomorphism $i: S \rightarrow G(S)$ satisfying the following universal property: If $H$ is a commutative group and $j: S \rightarrow H$ a semigroup homomorphism, then there exists a unique group homomorphism 
$h: G(S) \rightarrow H$ with $h \circ i=j$. Moreover, $i$ is injective if, and only if, $S$ is cancellative.

Consider the functions $l: S \rightarrow \mathbf{N} \cup\{\infty\}$ and $t: S \rightarrow \mathbf{N} \cup\{\infty\}$ given by

$$
l(\alpha):=\sup \left\{n \in \mathbf{N} \mid \alpha=\sum_{i=1}^{n} \alpha_{i}, \text { where } \alpha_{i} \in S \backslash\{0\}\right\}
$$

and

$$
t(\alpha):=\operatorname{card}\left\{\left\{\alpha_{i}\right\}_{i=1,2, \ldots, n} \text { finite subset of } S \backslash\{0\} \mid \alpha=\sum_{i=1}^{n} \alpha_{i}\right\} .
$$

It is clear that if $\alpha, \beta \in S$ then, $l(\alpha+\beta) \geq l(\alpha)+l(\beta)$, and also that $t(\alpha+\beta) \geq t(\alpha)+t(\beta)$.

Definition 1. A commutative semigroup with a zero element $S$ is said to be combinatorially finite (C.F.) if $t(\alpha)<\infty$ for each $\alpha$ in $S$.

Proposition 1. Let $S$ be a C.F. semigroup. Then the following statements hold.

i) For each $\alpha \in S$, there is no infinite sequence $\left\{\alpha_{i}\right\}_{i=1}^{\infty}$ of elements in $S \backslash\{0\}$ such that $\alpha-\sum_{i=1}^{n} \alpha_{i} \in S$ whenever $n \geq 1$.

ii) $S \cap(-S)=\{0\}$, where $-S=\{-x \in G(S) \mid x \in S\}$.

Proof.

i) If we had a sequence $\left\{\alpha_{i}\right\}_{i=1}^{\infty}$ as above, then $S$ would not be a C.F. semigroup since $t(\alpha)$ would equal $\infty$.

ii) Assume that there exists $\alpha \in S \cap(-S), \alpha \neq 0$. Write $\alpha_{i}=\alpha$ if $i$ is an even number and $\alpha_{i}=-\alpha$ whenever $\alpha$ is an odd number. Then, the sequence $\left\{\alpha_{i}\right\}_{i=1}^{\infty}$ contradicts i).

Corollary 1. Assume that $S$ is a C.F. semigroup, then:

i) $t(\alpha)=0$ if, and only if, $\alpha=0$.

ii) $l(\alpha)=0$ if, and only if, $\alpha=0$.

Proof. It is clear that $t(\alpha)$ and $l(\alpha)$ are not equal to 0 whenever $\alpha \neq 0$. Conversely, $t(0) \neq 0$ (or $l(0) \neq 0$ ) implies $0=\sum_{i=1}^{n} \alpha_{i}, \alpha_{i} \in S \backslash\{0\}, n \geq 2$ and therefore $S \cap(-S) \neq\{0\}$ which contradicts Proposition 1 .

Remark. Statement ii) in Proposition 1 allows us to prove the existence of a function $h: S \rightarrow \mathbf{N}$ satisfying $h(\alpha+\beta)=h(\alpha)+h(\beta)$ for $\alpha, \beta \in S$ and $h(\alpha)=0$ if, and only if, $\alpha=0$. When $S$ is finitely generated, the above condition implies $S$ combinatorially finite. As a consequence, both the existence of $h$ and statements i) and ii) in Proposition 1 can be taken as a definition of C.F. finitely generated semigroup.

On the other hand, we can not interchange the functions $t$ and $l$ in Definition 1 , since although $l(\alpha)<\infty$ for all $\alpha \in S$ holds whenever $S$ be a 
C.F. semigroup, the converse is not true. To see it, consider the additive semigroup $S=\mathbf{Z}_{1} \bigoplus \mathbf{Z}$, where $\mathbf{Z}_{1}=\{x \in \mathbf{Z} \mid x \geq 1\}$. Pick $\alpha=(x, y) \in S$, it is clear that the number of sums in a decomposition of $\alpha$ as a sum of elements in $S$ is $x$ or less. Therefore $l(\alpha)<\infty$. However, $S$ is not a C.F. semigroup, because, for instance, $(2,0)=(1, m)+(1,-m)$ for all $m \in \mathbf{Z}$.

Now, consider the value semigroup $S$ of a valuation. Next theorem gives some interesting properties of $S$.

Theorem 1. Let $v$ be a valuation of $F$ centered at $R$ and denote by $S(G)$ the value semigroup (group) of $v$. Then:

i) The groups $G(S)$ and $G$ are equal. Therefore $G(S)$ is ordered.

ii) $S$ is a cancellative ordered commutative semigroup which is torsion free.

iii) $S$ is a C.F. semigroup.

Proof.

i) $G$ contains $S$ and, since $F$ is the quotient field of $R$, we have $G \subseteq G(S)$. Therefore $G=G(S)$.

ii) Denote by $R_{v}=\{f \in F \backslash\{0\} \mid v(f) \geq 0\}$ the valuation ring of $v$. $R_{v}$ is a local ring and $m_{v}:=\{f \in F \backslash\{0\} \mid v(f)>0\}$ is its maximal ideal. Let $f \in F \backslash\{0\}$ be such that $v(f) \neq 0$. Then $v(f)$ (or $v(1 / f))>0$, so $f$ (or $1 / f) \in m_{v}$ and thus $f^{p}$ (or $\left.1 / f^{p}\right) \in m_{v}$ whenever $p \in \mathbf{N} \backslash\{0\}$. As a consequence, $v(f) \neq 0$ implies $v\left(f^{p}\right) \neq 0$. This proves that $G$ is a torsionfree group. Finally, all the properties of $S$ given in ii) are clear since $S$ is a subsemigroup of $G$.

iii) Recall that the Krull dimension of $R_{v}$ is usually called the rank of $v$ $(r k(v))$ and that a $v$-ideal of $R$ is the intersection of $R$ with an ideal of $R_{v} . R$ is a noetherian ring, therefore $r k(v)<\infty$ (see [6, App. 2]) and each $v$-ideal $a$ is spanned by finitely many elements in $R$, i.e., $a=\left\langle h_{1}, h_{2}, \ldots, h_{r}\right\rangle, h_{i} \in R$ $(1 \leq i \leq r)$. If $\alpha=\min \left\{v\left(h_{i}\right) \mid i=1,2, \ldots, r\right\}$, then it is straightforward that $a=P_{\alpha}:=\{f \in R \mid v(f) \geq \alpha\}$. So, the family $F=\left\{P_{\alpha}\right\}_{\alpha \in S}$ consists of all $v$-ideals of $R$.

To prove that $S$ is C.F., we first assume that $r k(v)=1$. Then $F$ forms a simple infinite descending chain under inclusion [6, Lemma 3, App. 3] and therefore, the elements in $S$ form a simple infinite ascending chain under the ordering in $S$. So $S$ is C.F. Now, apply induction on the rank of $v$ and assume that $S$ is not C.F. Then, we can express $\alpha=\alpha_{1 i}+\alpha_{2 i}, \alpha, \alpha_{1 i}, \alpha_{2 i} \in S$ and the sets $\left\{\alpha_{1 i}\right\}_{i=1}^{\infty}$ and $\left\{\alpha_{2 i}\right\}_{i=1}^{\infty}$ are infinite. $S$ is well-ordered since the set of $v$-ideals so is [6,App.3]. Consequently, rearranging the sets $\left\{\alpha_{1 i}\right\}_{i=1}^{\infty}$ and $\left\{\alpha_{2 i}\right\}_{i=1}^{\infty}$, we obtain that one of them constitutes a simple infinite descending chain. To show that this fact is not possible, we only need to observe that $v$ can be written $v=u \circ w$, where $u$ is of rank $r k(v)-1$ and $w$ is a rank one valuation of the residue field of $u$ and then, apply induction and the 
corollary of [6, App. 3], which asserts that if $b_{2} \subset b_{1}$ are two consecutive $u$-ideals, then the $v$-ideals $a$ such that $b_{2} \subset a \subset b_{1}$ are either finite in number or form a simple descending infinite sequence.

In the sequel, $S$ will denote the value semigroup of a valuation. An element $\alpha \in S$ is said to be irreducible if $l(\alpha)=1$. Then, we can state the following:

Corollary 2. The semigroup $S$ is generated by its irreducible elements. This set need not be finite.

Proof. The first statement is clear since $S$ is C.F. Now consider a valuation $v$ centered at a regular 2-dimensional noetherian local ring. Assume that the rank and the rational rank of $v$ equal 1 and that the transcendence degree of $v$ is 0 . Finally, suppose that the value group of $v$ is not isomorphic to $\mathbf{Z}$, then, $S$ has an infinite minimal system of generators. These generators are exactly the irreducible elements of $S$ which concludes the proof.

\section{The semigroup algebra of a valuation.}

Let $v$ be a valuation. Denote by $S$ its value semigroup. The semigroup algebra of $v$ is the semigroup $K$-algebra associated to $S$ and it will be denoted by $K[S] . K[S]$ is the $S$-graded $K$-algebra $K[S]=\bigoplus_{\alpha \in S}(K[S])_{\alpha},(K[S])_{\alpha}:=$ $K \alpha$.

Denote by $\Lambda$ a minimal set of generators of $S$ as semigroup. For instance, we can think of $\Lambda$ as the set of irreducible elements in $S . \Lambda$ is, in general, an infinite set. For a set $\mathbf{T}$, write $\mathbf{T}^{(\Lambda)}=\bigoplus_{\lambda \in \Lambda} \mathbf{T}_{\lambda}$ where $\mathbf{T}_{\lambda}=\mathbf{T}$. Consider the mapping $\psi: \mathbf{Z}^{(\Lambda)} \rightarrow G(S)$ given by $\psi\left(e_{\lambda}\right)=\lambda,\left\{e_{\lambda}\right\}_{\lambda \in \Lambda}$ being the standard basis of the $\mathbf{Z}$-module $\mathbf{Z}^{(\Lambda)}$. The ordering in $G(S)$ gives to $\mathbf{Z}^{(\Lambda)}$ an structure of lattice. The kernel of $\psi, L$, is a sublattice of $\mathbf{Z}^{(\Lambda)}$ whose intersection with $\mathbf{N}^{(\Lambda)}$ is the origin 0 . This can be easily deduced from the fact that $S \cap(-S)=\{0\}$. The morphism $\psi$ induces a surjective $K$ algebra homomorphism $\phi_{0}: K\left[\mathbf{N}^{(\Lambda)}\right] \rightarrow K[S]$ which allows to regard $K[S]$ as a $K\left[\mathbf{N}^{(\Lambda)}\right]$-module. We shall use two approaches to study the semigroup algebra of $v$. Firstly, we shall construct a minimal free resolution of the $K\left[\mathbf{N}^{(\Lambda)}\right]$-module $K[S]$ and we shall study its syzygy modules by means of a concrete simplicial complex and secondly, we shall obtain minimal free resolutions of the former module from certain type of cell complexes on the lattice module $M_{L}=K\left[\mathbf{N}^{(\Lambda)}+L\right] \subseteq K\left[\mathbf{Z}^{(\Lambda)}\right]$. In particular, we shall get a more explicit free resolution of $K[S]$.

\subsection{Syzygies of the semigroup algebra.}

3.1.1. . For a start, we state a basic result for the development of this subsection. It holds for semigroups $S$ satisfying $l(\alpha)<\infty$ for all nonzero 
element $\alpha \in S$. Thus, we can use it in our case: $S$ is the value semigroup of a valuation. Let $A$ be an $S$-graded ring $A=\bigoplus_{\alpha \in S} A_{\alpha}$ and $M=\bigoplus_{\alpha \in S} M_{\alpha}$ an $S$-graded $A$-module.

Proposition 2 (Graded Nakayama's Lemma). Let $A$ and $M$ be as above. Denote by $m=\bigoplus_{\alpha \in S, \alpha \neq 0} A_{\alpha}$ the irrelevant ideal of $A$. If $m M=M$, then $M=0$.

Proof. If $M \neq 0$, then there exists an element $\beta \in S$ such that the degree $\beta$ homogeneous component of $M, M_{\beta}$, does not vanish. Now $M_{\beta}=(m M)_{\beta}$ proves that $\beta$ can be written $\beta=\delta+\gamma ; \delta, \gamma \in S$ and $M_{\gamma} \neq 0$. Iterating, we conclude that $l(\beta)$ is not finite, which is a contradiction.

Now consider the $K$-algebra $K\left[\mathbf{N}^{(\Lambda)}\right]$ which, for the sake of simplicity, will be expressed as a polynomial ring $K\left[\left\{X_{\lambda}\right\}_{\lambda \in \Lambda}\right]$ with, possibly, infinitely many indeterminates and it will be denoted by $A_{v} . A_{v}$ is $S$-graded if we give degree $\lambda \in S$ to the indeterminate $X_{\lambda}$ and so, we can express $A_{v}=\bigoplus_{\alpha \in S}\left(A_{v}\right)_{\alpha}$, where $\left(A_{v}\right)_{\alpha}$ denotes the homogeneous component of degree $\alpha$ of $A_{v} .\left(A_{v}\right)_{\alpha}$ is a $K$-vector space. Note that, for any semigroup $S$, we have that $S$ is C.F. if, and only if, $\operatorname{dim}_{K}\left(A_{v}\right)_{\alpha}<\infty$ and $l(\alpha)<\infty$ for all $\alpha \in S$. Denote by $M_{v}$ the irrelevant ideal of $A_{v}$ and by $I_{0}$ the kernel of $\phi_{0} . I_{0}$ is a homogeneous ideal of $A_{v}$. Let $B$ be a minimal homogeneous generating set of $I_{0}$ and denote by $B_{\alpha}$ the set of elements in $B$ of degree $\alpha$. Applying Proposition 2, it is straightforward to deduce that the set of classes in $I_{0} / M_{v} I_{0}$ of the elements of $B_{\alpha}$ is a basis of the vector space of the homogeneous component of degree $\alpha$ of $I_{0} / M_{v} I_{0} . B_{\alpha}$ is a finite set since $\left(A_{v}\right)_{\alpha}$ is a finite-dimensional vector space. Set $B_{\alpha}=\left\{Q_{1}, Q_{2}, \ldots, Q_{d(\alpha)}\right\}$ and $L_{1}:=\bigoplus_{\alpha \in S}\left(A_{v}\right)^{d(\alpha)}$. If $\phi_{1, \alpha}:\left(A_{v}\right)^{d(\alpha)} \rightarrow A_{v}$ is the $A_{v}$-module homomorphism given by $\phi_{1, \alpha}\left(a_{1}, a_{2}, \ldots, a_{d(\alpha)}\right)=\sum_{i=1}^{d(\alpha)} a_{i} Q_{i}$, then we have the $A_{v}$-module homomorphism $\phi_{1}: L_{1} \rightarrow A_{v}, \phi_{1}=\sum_{\alpha \in S} \phi_{1, \alpha}$. We give degree $\alpha$ to the generators of $\left(A_{v}\right)^{d(\alpha)}$, thus $L_{1}$ is an $S$-graded free $A_{v}$-module and $\phi_{1}$ a homogeneous homomorphism of degree 0 . Repeating this procedure for each syzygy module $I_{i}:=\operatorname{Ker} \phi_{i}$, we get a minimal free resolution of the $S$-graded $A_{v}$-module $K[S]$ :

$$
\cdots \rightarrow L_{i} \stackrel{\phi_{i}}{\rightarrow} L_{i-1} \rightarrow \cdots \rightarrow L_{1} \stackrel{\phi_{1}}{\rightarrow} A_{v} \rightarrow K[S] \rightarrow 0 .
$$

Tensoring by $K$, we note that there exists a homogeneous degree 0 isomorphism of $S$-graded $A_{v}$-modules between the $i$-th Tor module $\operatorname{Tor}_{i}^{A_{v}}(K[S], K)$ and $L_{i} \bigotimes_{A_{v}} K, i \geq 0$.

On the other hand, we can consider a generalized Koszul complex as follows:

$$
\cdots \rightarrow \bigwedge^{p} A_{v}^{(\Lambda)} \stackrel{d_{p}}{\rightarrow} \bigwedge^{p-1} A_{v}^{(\Lambda)} \rightarrow \cdots \rightarrow A_{v}^{(\Lambda)} \stackrel{d_{1}}{\rightarrow} A_{v} \stackrel{d_{0}}{\rightarrow} K \rightarrow 0
$$


$d_{0}$ is the natural obvious epimorphism and if $\left\{e_{\lambda}\right\}_{\lambda \in \Lambda}$ is the standard basis of the $A_{v}$-module $A_{v}^{(\Lambda)}$, then we have

$$
d_{p}\left(e_{J}\right)=\sum_{r=1}^{p}(-1)^{r} X_{\lambda_{r}} e_{J \backslash\left\{\lambda_{r}\right\}},
$$

where $e_{J}=e_{\lambda_{1}} \wedge e_{\lambda_{2}} \wedge \cdots \wedge e_{\lambda_{p}}$ whenever $J=\left\{\lambda_{1}, \lambda_{2}, \ldots, \lambda_{p}\right\} \subseteq \Lambda . \wedge^{p} A_{v}^{(\Lambda)}$ can be regarded as an $S$-graded $A_{v}$-module by giving to $e_{J}$ the degree $\sum_{r=1}^{p} \lambda_{r}$. Thus (1) is an $S$-graded free resolution where all the homomorphisms are homogeneous of degree 0 .

We shall write $K[S] .(\Lambda)$ for the complex obtained by tensoring (1) through with $K[S]$ :

$$
\cdots \rightarrow \bigwedge^{p}(K[S])^{(\Lambda)} \stackrel{e_{p}}{\rightarrow} \bigwedge^{p-1}(K[S])^{(\Lambda)} \rightarrow \cdots \rightarrow K[S] \stackrel{e_{0}}{\rightarrow} K \bigotimes_{A_{v}} K[S] \rightarrow 0 .
$$

The formula for $e_{p}$ is the same one as $d_{p}$ but replacing $X_{\lambda_{r}}$ by $\lambda_{r}$. Furthermore the homomorphisms $e_{p}$ are homogeneous of degree 0 under the induced gradings. As a consequence, taking into account the commutative property of the Tor functor, there exists a homogeneous degree 0 isomorphism of $S$ graded $A_{v}$-modules between the $i$-th Tor module $\operatorname{Tor}_{i}^{A_{v}}(K, K[S])$ and the $i$-th homology module $H_{i}(K[S] .(\Lambda))$.

Finally, for each $\alpha \in S$, we give a $K$-vector space complex isomorphic to that of homogeneous components of degree $\alpha$ in $K[S]$. $(\Lambda)$. Denote by $P(\Lambda)$ the power set of $\Lambda, P(\Lambda)$ is an abstract simplicial complex. Set

$$
\triangle_{\alpha}:=\left\{J \subseteq \Lambda \mid J \text { is a finite subset of } \Lambda \text { and } \alpha-\sum_{J} \in S\right\},
$$

where $\sum_{J}=\sum_{\lambda \in J} \lambda . \triangle_{\alpha}$ is a simplicial subcomplex of $P(\Lambda)$. Associate to $\triangle_{\alpha}$, we consider the complex of vector spaces $C .\left(\triangle_{\alpha}\right)$ such that its vector spaces are $C_{i}\left(\triangle_{\alpha}\right)=\bigoplus_{J \in \triangle_{\alpha}, \operatorname{card}(J)=i+1} K J, i \geq-1$ and its boundaries $\partial: C_{i}\left(\triangle_{\alpha}\right) \rightarrow C_{i-1}\left(\triangle_{\alpha}\right)$ are given by $\partial(J)=\sum_{\beta \in J}(-1)^{\eta_{J}(\beta)} J \backslash\{\beta\}$, where $\eta_{J}(\beta)$ denotes the number of place that $\beta$ has among the elements in $J$. The homology of this complex will be called the augmented homology of $\triangle_{\alpha}$. This subsection can be summarized in the following:

Theorem 2. For each $\alpha \in S$, there exists an explicit isomorphism of $K$ vector spaces between the vector space $\left(I_{i}\right)_{\alpha} /\left(M_{v} I_{i}\right)_{\alpha}$ of $i$-th syzygies of degree $\alpha$ of $K[S]$ as $A_{v}$-module and the $i$-th augmented homology vector space of the simplicial complex $\triangle_{\alpha}, \widetilde{H}_{i}\left(\triangle_{\alpha}\right)$.

3.1.2. . We devote this subsection to show how bases for the homology $\widetilde{H}_{i}\left(\triangle_{\alpha}\right)$ can be explicitly computed from bases of the homology of vector space complexes associated to directed graphs which depend on the set $\Lambda$. 
This will be done adapting the results by Campillo and Gimenez in the case of toric affine varieties [4].

To start with, we describe the type of vector space complexes which we shall use to compute $\widetilde{H}_{i}\left(\triangle_{\alpha}\right)$. Assume that $\Gamma$ is a subset of $\Lambda$, which is a finite set of generators of a semigroup $T$, and $B$ a subset of $T$. We shall call the directed graph of $T$ associated to the pair $(\Gamma, B)$ to the directed graph $G_{\Gamma B}(T)$ (denoted $G_{\Gamma B}$ if it does not cause confusion) whose vertex set is $\left\{m \in T \mid m-\sum_{L} \in B\right.$ for some subset $\left.L \subseteq \Gamma\right\}$ and such that $\left(m, m^{\prime}\right)$ is an edge iff $m^{\prime}=m+\gamma$ for some $\gamma \in \Gamma$. A $K$-vector space complex $C .\left(G_{\Gamma B}(T, m)\right)$ can be associated to the pair $\left(G_{\Gamma B}, m\right), m$ being a vertex of $G_{\Gamma B}$, if the following condition holds: Whenever $b \in B$ and $\lambda, \lambda^{\prime} \in \Gamma$ satisfy $b+\lambda+\lambda^{\prime} \in B$, then $b+\lambda \in B$ and $b+\lambda^{\prime} \in B$. In such a case $G_{\Gamma B}$ is called to be a chain graph. Each vector space $C_{i}\left(G_{\Gamma B}(T, m)\right), i \geq-1$, is equal to $\bigoplus K L$ where the sum is over all subsets $L$ of $\Gamma$ of cardinality $i+1$ such that $m-\sum_{L} \in B$. The boundaries are induced by those of the simplicial complex $P(\Lambda)$.

Next, we state the main result of this subsection.

Theorem 3. The homology $\widetilde{H}_{i}\left(\triangle_{\alpha}\right)$ can be explicitly reached from finitely many homologies of $K$-vector space complexes of the type $C .\left(G_{\Gamma B}(T, m)\right)$ for suitable $T, \Gamma, B$ and $m$.

To reach a homology from others means to obtain bases of the homology from bases of the others by means of exact sequences. Let's see how to reach $\widetilde{H}_{i}\left(\triangle_{\alpha}\right)$. Let $\bar{S}_{\alpha}=\left\{\alpha^{\prime} \in S \mid \alpha-\alpha^{\prime} \in S\right\} . \bar{S}_{\alpha}$ is finite since $S$ is C.F. Denote by $S_{\alpha}$ the subsemigroup of $S$ spanned by $\bar{S}_{\alpha}$. It is not difficult to prove that $\triangle_{\alpha}=\left\{J \subseteq \bar{S}_{\alpha} \mid \alpha-\sum_{J} \in S_{\alpha}\right\}$. Now, pick a partition of $\bar{S}_{\alpha}, \bar{S}_{\alpha}=\Omega_{\alpha} \cup \Pi_{\alpha}$, consider the Apery set of $\bar{S}_{\alpha}$ relative to $\Pi_{\alpha}$ :

$$
A(\alpha)=A=\left\{a \in S_{\alpha} \mid a-e \notin S_{\alpha} \text { for all } e \in \Pi_{\alpha}\right\}
$$

and the related set

$$
\begin{aligned}
K_{\alpha}:=\left\{L \subseteq \bar{S}_{\alpha} \mid L \cap \Pi_{\alpha} \neq \emptyset \text { and } \alpha-\sum_{L}\right. & \left.\in S_{\alpha}\right\} \\
& \cup\left\{L \subseteq \Omega_{\alpha} \mid \alpha-\sum_{L} \in S_{\alpha} \backslash A\right\} .
\end{aligned}
$$

There is no loss of generality in assuming that $\alpha$ is a vertex of $G_{\Omega_{\alpha} A}\left(S_{\alpha}\right)$ and then, it is clear that the complex associate to $\left(G_{\Omega_{\alpha} A}, \alpha\right)$ makes sense. It will be denoted $C .(A(\alpha))$ and it is exactly the augmented relative simplicial complex $\widetilde{C} \cdot\left(\triangle_{\alpha}, K_{\alpha}\right)$. Therefore, we can state the following long exact sequence, which allows to reach the homology $\widetilde{H}_{i}\left(\triangle_{\alpha}\right)$ from others.

$$
\cdots \rightarrow H_{i+1}\left(A_{\alpha}\right) \rightarrow \widetilde{H}_{i}\left(K_{\alpha}\right) \rightarrow \widetilde{H}_{i}\left(\triangle_{\alpha}\right) \rightarrow H_{i}\left(A_{\alpha}\right) \rightarrow \widetilde{H}_{i-1}\left(K_{\alpha}\right) \rightarrow \ldots
$$


$H_{i+1}\left(A_{\alpha}\right)$ and $H_{i}\left(A_{\alpha}\right)$ are as we desire. Let us see that $\widetilde{H}_{i}\left(K_{\alpha}\right)$ and $\widetilde{H}_{i-1}\left(K_{\alpha}\right)$ so are. Firstly, define the simplicial complex

$$
\begin{array}{r}
\bar{K}_{\alpha}:=K_{\alpha} \cup\left\{L=I \cup J \mid I \subseteq \Omega_{\alpha}, J \subseteq \Pi_{\alpha}, \operatorname{card}(J) \geq 2, \alpha-\sum_{I \cup J} \notin S_{\alpha}\right. \\
\text { but } \left.\alpha-\sum_{I}-e \in S_{\alpha} \text { for each } e \in J\right\}
\end{array}
$$

and the subcomplexes of $\bar{K}_{\alpha}$,

$$
K_{\alpha}(j):=K_{\alpha} \cup\left\{L=I \cup J \in \bar{K}_{\alpha} \backslash K_{\alpha} \mid \operatorname{card}(J) \leq j\right\},
$$

$1 \leq j \leq \operatorname{card}\left(\Pi_{\alpha}\right)$. $\quad \bar{K}_{\alpha}$ is acyclic and so $\widetilde{H}_{i+1}\left(\bar{K}_{\alpha}, K_{\alpha}\right) \cong \widetilde{H}_{i}\left(K_{\alpha}\right)$. Also $\widetilde{H}_{i}\left(\bar{K}_{\alpha}, K_{\alpha}\right) \cong \widetilde{H}_{i}\left(K_{\alpha}\left(\operatorname{card}\left(\Pi_{\alpha}\right)\right), K_{\alpha}(1)\right)$. This last homology can be reached from $\widetilde{H}_{i}\left(K_{\alpha}(j), K_{\alpha}(j-1)\right), 2 \leq j \leq \operatorname{card}\left(\Pi_{\alpha}\right)$, since the following exact sequence of vector space complexes

$$
0 \rightarrow C .\left(K_{\alpha}(j), K_{\alpha}(i)\right) \rightarrow C .\left(K_{\alpha}(k), K_{\alpha}(i)\right) \rightarrow C .\left(K_{\alpha}(k), K_{\alpha}(j)\right) \rightarrow 0
$$

holds for sequences $(i, j, k)$ equal to $(1,2,3),(1,3,4), \ldots,\left(1, \operatorname{card}\left(\Pi_{\alpha}\right)-1\right.$, card $\left.\left(\Pi_{\alpha}\right)\right)$. As a consequence, we only need to show that the homology $\widetilde{H}_{i}\left(K_{\alpha}(j), K_{\alpha}(j-1)\right)$ can be computed from finitely many homologies of complexes associated to chain graphs. Indeed, a subset $J \subseteq \Pi_{\alpha}$ with card $(J) \geq 2$ is said to be associated to $d \in S_{\alpha}$, if $d-\sum_{J} \notin S_{\alpha}$ but $d-e \in S_{\alpha}$ for each $e \in J$. If we denote by $D_{\alpha}^{J}$ the set of elements $d$ in $S_{\alpha}$ such that $J$ is associated to $d$, then

$$
\widetilde{H}_{i}\left(K_{\alpha}(j), K_{\alpha}(j-1)\right) \cong \bigoplus_{J \subseteq \Pi_{\alpha}, \operatorname{card}(J)=j} H_{i-j}\left(G_{\Omega_{\alpha} D_{\alpha}^{J}}\left(S_{\alpha}, \alpha\right)\right) .
$$

A further study leads us to obtain finite subsets of $S_{\alpha}$, such that $\widetilde{H}_{i}\left(\triangle_{\alpha}\right)$ vanishes when $\alpha$ does not belong to them. In fact, for $-1 \leq l \leq \operatorname{card}\left(\Omega_{\alpha}\right)$ write

$$
M_{\alpha}(l):=K_{\alpha} \cup\left\{L=I \cup J \in \bar{K}_{\alpha} \backslash K_{\alpha} \mid \operatorname{card}(I) \leq l\right\} .
$$

As above,

$$
\widetilde{H}_{i}\left(\bar{K}_{\alpha}, K_{\alpha}\right) \cong \widetilde{H}_{i}\left(M_{\alpha}\left(\operatorname{card}\left(\Omega_{\alpha}\right)\right), M_{\alpha}(-1)\right) .
$$

This last homology can be reached from $\widetilde{H}_{i}\left(M_{\alpha}(l), M_{\alpha}(l-1)\right)$ and

$$
\widetilde{H}_{i}\left(M_{\alpha}(l), M_{\alpha}(l-1)\right) \cong \bigoplus \widetilde{H}_{i-l}\left(\Theta_{\alpha-\sum_{I}}\right),
$$

where the sum is over all subsets $I \subseteq \Omega_{\alpha}$ such that card $(I)=l$ and $\alpha-\sum_{I} \in$ $S_{\alpha}$, and where $\Theta_{d}=\left\{J \subseteq \Pi_{\alpha} \mid d-\sum_{J} \in S_{\alpha}\right\}$. Consequently, (2) and (3) 
prove that if we consider

$$
\begin{gathered}
C_{i}(\alpha):=\left\{m \in S_{\alpha} \mid m=a+\sum_{I} ; a \in A(\alpha), I \subseteq \Omega_{\alpha} \text { and } \operatorname{card}(I)=i+1\right\} \\
\cup\left\{m \in S_{\alpha} \mid \exists I \subseteq \Omega_{\alpha}, \operatorname{card}(I)=l \leq i \text { with } \widetilde{H}_{i-l}\left(\Theta_{m-\sum_{I}}\right) \neq 0\right\},
\end{gathered}
$$

then $\widetilde{H}_{i}\left(\triangle_{\alpha}\right)=0$ if $\alpha \notin C_{i}(\alpha)$. The simplicity of the set $\Theta_{d}$ has an important consequence:

Proposition 3 (See [4, Pr. 6.2]). The set $C_{i}(\alpha)$ is finite when we choose a suitable partition of the set $\bar{S}_{\alpha}$.

A crucial fact in the above proposition is that $S_{\alpha}$ is finitely generated. A suitable partition of $\bar{S}_{\alpha}$ would be a convex partition, that is, a partition $\bar{S}_{\alpha}=\Omega_{\alpha} \cup \Pi_{\alpha}$ where the cone generated by $S_{\alpha}\left(\right.$ in $\left.V_{S \alpha}:=G\left(S_{\alpha}\right) \bigotimes_{\mathbf{Z}} \mathbf{Q}\right)$ is equal to the cone generated by $\Omega_{\alpha}$ (in $V_{S \alpha}$ ) and card $\left(\Omega_{\alpha}\right)$ equals to the number of extremal rays of the cone spanned by $S_{\alpha}$.

3.2. The defining ideal of the semigroup. The $K$-algebra $K[S]$ is isomorphic to $A_{v} / I_{0}$. The ideal $I_{0}$, usually called the defining ideal of $S$, is spanned by a set of binomials which are difference of two monomials of the same degree. This set need not be finite. In the first part of this subsection, we shall use [2] to give a method to compute a minimal homogeneous generating set of $I_{0}, B$, formed by binomials of the type described above. This method uses the structure of graph of the simplicial complex $\triangle_{\alpha}$. On the other hand, denote by $L_{v}=K\left[\left\{X_{\lambda}^{ \pm 1}\right\}_{\lambda \in \Lambda}\right]$ the Laurent polynomial ring associate to the set $\Lambda$ and write $X^{a}=\prod_{\lambda \in \Lambda^{\prime}} X_{\lambda}^{a_{\lambda}} \in L_{v}$ whenever $a=\sum_{\lambda \in \Lambda^{\prime}} a_{\lambda} e_{\lambda} \in \mathbf{Z}^{(\Lambda)}, \Lambda^{\prime}$ being a finite subset of $\Lambda$. Obviously, $A_{v} \subset L_{v}=K\left[\mathbf{Z}^{(\Lambda)}\right]$. Recalling the notation at the beginning of Section 3, we observe that

$$
I_{0}=\left\langle X^{a}-X^{b} \mid a-b \in L\right\rangle \subset A_{v} .
$$

Following the ideas of $[\mathbf{1}]$, this fact will serve us, in the second part of this subsection, to obtain minimal free resolutions of $K[S]$ as $A_{v}$-module from suitable cell complexes on $M_{L}$.

3.2.1. Minimal generating sets of the defining ideal. A minimal homogeneous generating set of $I_{0}, B$, can be expressed $B=\cup_{\alpha \in S} B_{\alpha}$, where $B_{\alpha}$ is the set of elements in $B$ of degree $\alpha$. As a consequence of 3.1.1, we have that $B_{\alpha}$ is a finite set and card $B_{\alpha}=\operatorname{dim}_{K} \widetilde{H}_{0}\left(\triangle_{\alpha}\right)$. Moreover, $\triangle_{\alpha}$ is a graph which has $\operatorname{dim}_{K} \widetilde{H}_{0}\left(\triangle_{\alpha}\right)+1$ connected components. If $a=\sum_{\lambda \in \Lambda^{\prime}} a_{\lambda} e_{\lambda} \in \mathbf{N}^{(\Lambda)}\left(a_{\lambda} \neq 0\right)$, then $X^{a} \in A_{v}$, the support of $X^{a}$, $\operatorname{Supp}\left(X^{a}\right)$, is the set $\Lambda^{\prime}$ and the degree of $X^{a}, \operatorname{deg}\left(X^{a}\right)$, is $\sum_{\lambda \in \Lambda^{\prime}} a_{\lambda} \lambda \in S$.

It is clear that $I_{0}$ is an ideal generated by the set of binomials $\mathcal{B}=$ $\left\{X^{a}-X^{b} \mid \operatorname{deg}\left(X^{a}\right)=\operatorname{deg}\left(X^{b}\right)\right\}$. Let $C$ be a subset of $\mathcal{B}$ whose binomials 
have a fixed degree $\alpha$. We shall call graph associated to $C$ to a graph whose vertex set is the set of connected components of $\triangle_{\alpha}$ which contain the support of a monomial belonging to a binomial in $C$. Two connected components, those associated to the monomials $X^{a}$ and $X^{b}$, are adjacent by an edge whenever $X^{a}-X^{b} \in C$. $C$ will be a generating tree for $\triangle_{\alpha}$ if the graph associated to $C$ is, in fact, a tree.

Theorem 4. A subset $B=\cup_{\alpha \in S} B_{\alpha} \subseteq \mathcal{B}$ is a minimal homogeneous generating set of $I_{0}$ if, and only if, $B_{\alpha}$ is a generating tree for $\triangle_{\alpha}$ whenever $\operatorname{dim}_{K} \widetilde{H}_{0}\left(\triangle_{\alpha}\right) \neq 0$ and $B_{\alpha}=\emptyset$, otherwise.

This theorem is analogous to the stated in [2] for finitely generated semigroups and the proof runs similarly. It is based on the fact that two monomials $M$ and $M^{\prime}$ of degree $\alpha \in S$ satisfy $M-M^{\prime} \in\left(M_{v} I_{0}\right)_{\alpha}$ if, and only if, $\operatorname{Supp}(M)$ and $\operatorname{Supp}\left(M^{\prime}\right)$ are in the same connected component of $\triangle_{\alpha}$. Furthermore, it is possible to decide whether $\operatorname{dim}_{K} \widetilde{H}_{0}\left(\triangle_{\alpha}\right) \neq 0$ by a close method to that given in [2, Th. 3.11].

3.2.2. Cellular resolutions of $\boldsymbol{K}[\boldsymbol{S}]$. For a start, we establish a relation between the module $M_{L}=K\left[\mathbf{N}^{(\Lambda)}+L\right]$ and the semigroup algebra of $v$, $K[S]$. Denote by $A_{v}[L]$ the group algebra of $L$ over $A_{v} . A_{v}[L]$ is the subalgebra of $K\left[\left\{X_{\lambda}\right\}_{\lambda \in \Lambda},\left\{Z_{\lambda}^{ \pm 1}\right\}_{\lambda \in \Lambda}\right]$ generated by the monomials $X^{a} Z^{l}$ where $a \in \mathbf{N}^{(\Lambda)}$ and $l \in L$. Thus, we can give a $\mathbf{Z}^{(\Lambda)}$-grading on $A_{v}[L]$ by writing $\operatorname{deg}\left(X^{a} Z^{l}\right)=a+l$. On the other hand, the morphism $h: A_{v}[L] \rightarrow M_{L}$, $X^{a} Z^{l} \rightarrow X^{a+l}$ gives to $M_{L}$ an structure of $\mathbf{Z}^{(\Lambda)}$-graded $A_{v}[L]$-module. Moreover, if $J=\operatorname{Ker}(h)$, then the following equality chain holds,

$$
M_{L} \bigotimes_{A_{v}[L]} A_{v}=A_{v}[L] / J \bigotimes_{A_{v}[L]} A_{v}=A_{v} / I_{0}=K[S] .
$$

Next, we shall consider two equivalent categories $\mathcal{A}$ and $\mathcal{B}$. $\mathcal{A}$ contains $M_{L}$, and $K[S]$, viewed as $A_{v}$-module, is in $\mathcal{B}$. This shall give the desired relation between $M_{L}$ and $K[S] . \mathcal{A}$ will be the category of $\mathbf{Z}^{(\Lambda)}$-graded $A_{v}[L]$ modules, where the morphisms are $\mathbf{Z}^{(\Lambda)}$-graded $A_{v}[L]$-module homomorphisms of degree 0 , and $\mathcal{B}$ the category of $G(S)$-graded $A_{v}$-modules, where the morphisms are, also, of degree 0 . Note that $K[S]$ is $S$-graded and therefore $G(S)$-graded. The functor $\pi: \mathcal{A} \rightarrow \mathcal{B}$ which gives the equivalence is $\pi(M)=M \bigotimes_{A_{v}[L]} A_{v}$. Notice that if $M \in \mathcal{A}, M=\bigoplus_{a \in \mathbf{Z}^{(\Lambda)}} M_{a}$, then $\pi$ identifies as $\pi(M)_{\alpha}, \alpha \in G(S)$, all the vector spaces $M_{a}$ such that $\psi(a)=\alpha$, where $\psi$ is the mapping given at the beginning of Section 3. A complete proof of this equivalence is similar to that of the case of finitely generated semigroups [1, Th. 3.2] and we omit it.

Now, taking into account that the degrees of $M_{L}$ are in $\mathbf{N}^{(\Lambda)}+L$, we can state: 
Theorem 5. Let $\pi: \mathcal{A} \rightarrow \mathcal{B}$ be the equivalence of categories above given. Then $\pi$ transforms $\mathbf{Z}^{(\Lambda)}$-graded (minimal) free resolutions of $M_{L}$ as $A_{v}[L]$ module into $S$-graded (minimal) free resolutions of $K[S]$ as $A_{v}$-module, and conversely.

Finally, we shall see how to get free resolutions of $M_{L}$ from regular cell complexes and, consequently, how to get free resolutions of $K[S]$. First at all, denote by $\leq$ the ordering in $\mathbf{Z}^{(\Lambda)}$ defined so: $a \leq b$ if, and only if, $b-a \in \mathbf{N}^{(\Lambda)}$. Also, set $\min \left(M_{L}\right):=\left\{X^{a} \in M_{L} \mid X^{a} / X_{\lambda} \notin M_{L}\right.$ for all $\left.\lambda \in \Lambda\right\}$.

Proposition 4. The $\mathbf{Z}^{(\Lambda)}$-graded $A_{v}$-module $M_{L}$ satisfies the following properties:

i) The set of monomials in $M_{L}$ of degree $\leq a$ is finite for each $a \in \mathbf{Z}^{(\Lambda)}$.

ii) $M_{L}$ is generated as $A_{v}$-module by the set $\min \left(M_{L}\right)$.

Proof.

i) Write $a=\sum_{\lambda \in \Lambda^{\prime} \subset \Lambda} a_{\lambda} e_{\lambda}$ and set $a^{+}=\sum_{\lambda \in \Lambda^{\prime}, a_{\lambda}>0} a_{\lambda} e_{\lambda}$ and $a^{-}=$ $\sum_{\lambda \in \Lambda^{\prime}, a_{\lambda}<0} a_{\lambda} e_{\lambda}$. If $d$ is the degree of a monomial in $M_{L}$, then $d=l+b^{+}$, where $l \in L$ and $b^{+} \in \mathbf{N}^{(\Lambda)}$. It is clear that, as above, $l=l^{+}+l^{-}$where $\psi\left(l^{+}\right)=-\psi\left(l^{-}\right) \in S$. So, $d \leq a$ if, and only if, $l^{+}+b^{+}+l^{-} \leq a^{+}+a^{-}$. As a consequence the set $\left\{l^{+} \mid d \leq a\right\}$ is finite and so is the set $\left\{\psi\left(l^{+}\right) \mid d \leq a\right\} \subseteq S$. Finally, $\left\{l^{-} \mid d \leq a\right\}$ is also a finite set, since $S$ is a C.F. semigroup.

ii) This is a straightforward consequence of the fact that, there is no infinite decreasing sequence under divisibility of monomials in $M_{L}$, which follows from i).

Put $\min \left(M_{L}\right)=\left\{X^{a} \mid a \in I \subset \mathbf{Z}^{(\Lambda)}\right\} . \quad I$ is, generally, an infinite set. Consider a regular cell complex $X$ such that $I$ is its set of vertices and $\epsilon$ an incidence function on pairs of faces. A typical example of a regular cell complex is the set of faces of a convex polytope.

Associated to $X$, a cellular complex of $A_{v}$-modules $M .(X)$ can be defined in the following way: The modules are $M_{i}(X)=\bigoplus_{J \in X, \operatorname{dim} J=i} A_{v} J, i \geq 0$, (we have identified the face $J$ in $X$ with its set of vertices) and the boundaries are given by

$$
\partial J=\sum_{J^{\prime} \in X, J^{\prime} \neq \emptyset} \epsilon\left(J, J^{\prime}\right)\left(m_{J} / m_{J^{\prime}}\right) J^{\prime}
$$

where $m_{J}$ is the least common multiple of the set $\left\{X^{a} \mid a \in J\right\}$. M. $(X)$ is $\mathbf{Z}^{(\Lambda)}$-graded, the degree of a face $J$ being the exponent vector of $m_{J}$. When $M .(X)$ is a free resolution of $M_{L}$, it is called to be a cellular resolution of $M_{L}$. Set $\triangle=\{J \in P(I) \mid J$ is a finite set $\}$ and associate to $\triangle$ an incidence function as in the definition of $\triangle_{\alpha}$ (see 3.1.1). $\triangle$ is a cell complex and its associated cellular complex $M .(\triangle)$ is a cellular resolution of $M_{L}$ called the Taylor resolution of $M_{L}$. This is an easy consequence of the fact that 
the subcomplex $\triangle_{\leq a}$ of $\triangle$ on the vertices of degree $\leq a$ is acyclic for all $a \in \mathbf{N}^{(\Lambda)}$.

We desire to apply Theorem 5 to get free resolutions of $K[S]$. In order to do it, we observe that the mapping $\bigoplus_{J \in \mathcal{R}} A_{v}[L] J \rightarrow M_{i}(X), Z^{l} J \rightarrow J+l$ is an isomorphism of $\mathbf{Z}^{(\Lambda)}$-graded $A_{v}$-modules if $X$ satisfies that

$$
J+l \in X \text { whenever } J \in X \text { and } l \in L,
$$

$\mathcal{R}$ being a set of representatives of the set of $i$-dimensional orbits defined by the action of $L$ over $X$. Thus, we shall call to $X$ equivariant if it satisfies (5) and $\epsilon\left(J, J^{\prime}\right)=\epsilon\left(J+l, J^{\prime}+l\right)$ for all $l \in L$. If $X$ is equivariant, it is straightforward that $M .(X)$ is a $\mathbf{Z}^{(\Lambda)}$-graded complex of $A_{v}[L]$-modules and that $M .(X)$ is exact over $A_{v}$ if, and only if, it is exact over $A_{v}[L]$. In this case, $M .(X)$ is called an equivariant cellular resolution of $M_{L}$. Applying Theorem 5, we have proved the following:

Theorem 6. Let $S$ be the value semigroup of a valuation. If $M .(X)$ is a (minimal) equivariant cellular resolution of $M_{L}$, then $\pi(M .(X))$ is a (minimal) free resolution of $K[S]$ as $A_{v}$-module.

$\triangle$ is an equivariant cell complex. Its simplicity allows us to give an explicit resolution of $K[S]$ as $A_{v}$-module. For each $\alpha \in S$, denote by $\operatorname{mon}\left(A_{v}\right)_{\alpha}$ the set of monomials in $\left(A_{v}\right)_{\alpha}$ and by $E_{i}(\alpha)$ the set of cardinality $i$ subsets of $\operatorname{mon}\left(A_{v}\right)_{\alpha}$ whose greatest common divisor is 1 . Now, if $F_{i}(a)$ denotes the set of cardinality $i$ subsets of $\min \left(M_{L}\right)$ whose least common multiple is $a \in \mathbf{Z}^{(\Lambda)}$, it is clear, from the definition of $M .(\triangle)$, that $M_{i}(\triangle)=\bigoplus_{a \in \mathbf{N}^{(\Lambda)}+L} A_{v} F_{i}(a)$.

Regarding $M_{i}(\triangle)$ as $A_{v}[L]$-module and by Theorem 5 , it is clear that $\pi$ takes $F_{i}(a)$ bijectively to $E_{i}(\psi(a)), \pi(J)=\left\{X^{a} / X^{c} \mid X^{c} \in J\right\}$. As a consequence $\pi(M .(\triangle))$ can be expressed so: The $A_{v}$-modules are $\bigoplus_{\alpha \in S} A_{v} E_{i}(\alpha)$ and the boundaries are given by

$$
\partial(I)=\sum_{X^{c} \in I}(-1)^{\eta_{I}\left(X^{c}\right)} \operatorname{gcd}\left(I \backslash\left\{X^{c}\right\}\right)\left[I \backslash\left\{X^{c}\right\}\right],
$$

where $I \in E_{i}(\alpha), \eta_{I}$ is defined as in 3.1.1 and $\left[I \backslash\left\{X^{c}\right\}\right]$ means to remove the common factor $\operatorname{gcd}\left(I \backslash\left\{X^{c}\right\}\right)$ from $I \backslash\left\{X^{c}\right\}$.

\section{References}

[1] D. Bayer and B. Sturmfels, Cellular resolutions of monomial modules, J. Reine Angew. Math., 502 (1998), 123-140, MR 99g:13018, Zbl 0909.13011.

[2] E. Briales, A. Campillo, C. Marijuan and P. Pison, Minimal systems of generators for ideals semigroups, J. Pure Appl. Algebra, 124 (1998), 7-30, MR 98k:20105, Zbl 0913.20036.

[3] W. Bruns and J. Herzog, Cohen-Macaulay Rings, Cambridge University Press, Cambridge (1993), MR 95h:13020, Zbl 0788.13005. 
[4] A. Campillo and P. Gimenez, Syzygies of affine toric varieties, J. Algebra, 225(1) (2000), 142-165, MR 2001j:13014, Zbl 0973.14027.

[5] A. Campillo and C. Marijuan, Higher order relations for a numerical semigroup, Sem. Theorie des Nombres, Bordeaux, 3 (1991), 249-260, MR 93d:13027, Zbl 0818.20078.

[6] O. Zariski and P. Samuel, Commutative Algebra, Vol. II, Springer-Verlag (1960), MR 22 \#11006, Zbl 0121.27801.

Received September 25, 2000. The author was supported by DGICYT BFM2001-2251 and by F. Bancaixa 1I205.01/1.

D. Matemáticas (ESTCE)

UJI, Campus Riu SEC.

12071 CAStellón. SpAin

E-mail address: galindo@nuvol.uji.es 